\title{
DENTAL STEM CELLS (CONCEPTS AND APPLICATIONS)
}

\author{
Ahmed M. Hussein ${ }^{1 *} P h D$, Zeinab E. Darwish ${ }^{2} P h D$, Hanaa S. Raslan ${ }^{3} P h D$, \\ Mahmoud A. Attia ${ }^{4} P h D$, Hend M. Abdel-Hamid ${ }^{5} P h D$.
}

\section{ABSTRACT}

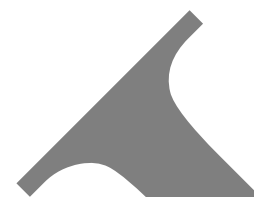

INTRODUCTION: Stem cells constitute the source of differentiated cells for the generation of tissues during development, as well as for regeneration of tissues that are diseased or injured postnatally. The stem cell research has grown exponentially to improve the life of patients with conditions that span from Alzheimer's disease, cardiac ischemia to bone or tooth loss. In dentistry, stem cell biology and tissue engineering are of great interest since they may provide an innovative for generation of clinical material and/or tissue regeneration. Mesenchymal stem cells were demonstrated in dental tissues, including dental pulp, periodontal ligament, dental papilla, and dental follicle. These stem cells can be isolated and grown under defined tissue culture conditions and are potential cells for use in tissue engineering including dental tissue, nerves and bone regeneration under appropriate conditions. This review was performed to study the concepts of stem cells and the most recent application of dental stem cells. KEY WORDS: Stem cells, Dental stem cells, Pluripotent, Multipotent. RUNNING TITLE: Dental Stem Cells.

1. Lecturer of Oral and Maxillofacial Pathology Department, Faculty of Oral and Dental Medicine, SVU, Qena, Egypt.

2. Professor of Oral Pathology, Faculty of Dentistry, Alexandria University, Alexandria, Egypt.

3. Vice Dean of Student's Affair, Professor of Oral Pathology, Faculty of Dentistry, Alexandria University, Alexandria, Egypt.

4. Assistant Professor of Oral Pathology, Department of Oral Pathology, Faculty of Dental Medicine, Al-Azhar University, Assiut Branch, Egypt.

5. Lecturer of Oral Pathology, Faculty of Dentistry, Alexandria University, Alexandria, Egypt

* Corresponding Author:

E-mail aattay1@gmail.com.

\section{INTRODUCTION}

Stem cells are very unique cells which have an ability to develop into several distinct cell types; aiding as a repair system for the body. When a stem cell divides, each cell has the potential to either remain a stem cell or become another type of cell with a more specialized function (i.e. a muscle cell, a red blood cell, a brain cell ...etc.). All stem cells have the same three general properties as self-renewal, unspecialization and unlimited potency (1).

\section{Stem cell classification according to their potency into}

- Totipotent stem cells are produced by the first few divisions of the fertilized egg. Early (1-3 days) embryo is totipotent. These cells can differentiate into embryonic and extra embryonic cell types.

- Pluripotent stem cells (PSCs) are the descendants of totipotent cells and can differentiate into cells derived from the 3 germ layers. These cells are derived from the inner cell mass of blastocyst (5 to 14 days).

- Multipotent stem cells can produce only cells of a closely related family of cells (e.g. hematopoietic stem cells differentiate into red blood cells, white blood cells,

- Unipotent stem cells can produce only one cell type, but have the property of self-renewal which distinguishes them from non-stem cells (2).
Stem cells can also be categorized as embryogenic stem cells (ESCs) or adult stem cells according to the stage of isolation. Embryogenic stem cells are totipotent. Although they have the greatest biological potential, ethical issues on the use of ESCs has precluded their widespread study, especially in humans. The focus has moved towards adult stem cells, which are derived from postnatal fully developed tissue and they are thought to renew cell populations, maintain tissue homeostasis and participate in tissue repair following injury. Compared with ESCs, adult derived stem cells have several limitations with respect to lifespan and differentiation potential (2).

It was found that induced pluripotent stem cells (iPSCs) were developed. These are reprogrammed somatic cells having pluripotency like ESCs. This might provide an alternative pathway which eliminate the ethical issues regarding the use of tissue from human embryos and allow to overcome problems of rejection after non-autologous cell implantation. Therefore, they were expected to become the important tool in the advancement of personalized medicine. Basically, iPSCs have been generated by reprograming cells via incorporation of several genes and they have similarities to human ESCs in their morphologies, gene expression, in-vitro differentiation potential and teratoma formation (3). 
Based on stem cell ability to rescue and/or repair injured tissue and partially restore organ function, multiple types of stem/progenitor cells have been speculated. Growing evidences demonstrated that stem cells are primarily found in niches and that certain tissues contain more stem cells than others. Dental tissues are considered a rich source of mesenchymal stem cells (MSCs) that are suitable for tissue engineering applications. It is known that these stem cells have the potential to differentiate into several cell types including odontoblasts, neural progenitors, osteoblasts, chondrocytes and adipocytes (4).

Oral tissues have been considered a potential source of multipotent stem cells like populations. With the exception of enamel, which lacks ameloblasts or other cellular elements following tooth development. The periodontium and dentine continue to retain some regenerative or reparative capacities. Dental multipotent stem cells are indicated for the regeneration of the dentin-pulp complex, bone, cartilage and neuronal tissues (5). Different populations have been isolated and characterized in postnatal dental tissues and classified according to the tissue of origin into:

1- Dental pulp stem cells (DPSCs) are ectomesenchymal multipotent stem cells. These cells have advantages for clinical applications when compared to other MSCs derived from bone marrow, adipose tissue, peripheral blood and umbilical cord blood. They are easily available from discarded teeth after extraction. The differentiation potential of DPSCs from natal teeth to adipogenic, osteogenic, chondrogenic, myogenic and neural-glial cell lines was also shown and an intrinsic tendency of these cells to differentiate towards osteoblasts has been demonstrated (6). Sometimes DPSCs are referred to as odontoblastoid cells, because they appear to synthesize and secrete dentin matrix like the odontoblasts that they replace. When transplanted in vivo the cells derived from dental pulp generated functional dental tissues similar to those generated by the cells of the dentin/pulp complexes. When cultured in ceramic substrates, such as hydroxyapatite or tricalcium phosphate; the cells are able to form bone, dentin and cementum like tissues (7).

2- Stem cells from exfoliated deciduous teeth (SCED) are a heterogeneous multicellular population of stem cells identified as highly proliferative clonogenic cells capable of differentiating into a variety of cell types including neural cells, adipocytes and odontoblasts. The SCED represent a population of multipotent stem cells which exhibited higher proliferation rates, increased population doublings, osteo-inductive capacity and ability to form sphere like clusters (8). On the other hand, immature dental pulp stem cells can be extracted from the pulp of primary teeth. They co-express mesenchymal and embryonic stem cell markers and present the capacity to differentiate into derivative cells of the 3 germinal layers. These cells can differentiation into smooth and skeletal muscles, neurons, cartilage, and bone under chemically defined culture conditions (9).

3- The cells located in the apical papilla (root foramen area) represent another unique population of dental stem cells. These cells show an ability to differentiate into cells of the osteogenic, odontogenic, adipogenic and neurogenic lineages. It is thought that stem cells from apical papilla

Alexandria Dental Journal. Volume 46 Issue 1 Section A may be responsible for the formation of primary odontoblasts that account for the formation of root dentin, whereas DPSCs seem to be the source of replacing odontoblasts that produce reparative dentine. They are derived from a developing tissue that may represent a population of early stem/progenitor cells, since they also showed other favorable characteristics, such as telomerase activity and improved migration capacity (8).

4-The periodontal ligament provides nourishment to the teeth, regulates periodontal homeostasis and contains a population of progenitor cells. Periodontal ligament stem cells (PDLSCs) are capable of differentiating into cells resembling cementoblasts, osteoblasts, adipocytes, chondrocytes, and fibroblasts (4). This population has a faster cell growth rate and higher clonogenic capability than bone marrow MSCs. These cells maintain their tissue regenerative potential even after recovery from frozen human tissue, which suggests the possibility of cryopreserved PDLSCs from extracted teeth being used for future therapeutic purposes (5). Periosteum derived stem cells lie in the thinner membrane of the periosteum. They undergo preferential osteogenic differentiation and possess mesenchymal multipotentiality. They can differentiate into osteoblasts, adipocytes and chondrocytes. But, they have limited potential for cell differentiation (10)

5- Dental follicle is a loose connective tissue sac surrounding the enamel organ and the dental papilla of the developing tooth germ before eruption. The stem cells were first isolated from the follicle of human impacted third molars. In vitro, dental follicle precursor cells have been demonstrating osteogenic, odontogenic and cementogenic differentiation capacities, as well as the tooth germ progenitor cells in the mesenchyme of the third molar tooth germ, can possess very high proliferative activity (11).

6-Gingiva represent a source of stem cell. Progenitor cells and multipotent stem cells subpopulations have been isolated from gingival fibroblasts. These fibroblasts are easily accessible and have used to induce pluripotent stem lines (6). Bone marrow stem cells are derived from mandible/maxilla. They have lower odontogenic potential than DPSCs. Safe cell expansion techniques are to be used (12). Epithelial stem cells develop from third molars of child. They are promising for tooth formation/regeneration. Their clinical application is difficult as it requires tooth donation from children (13). Adult human cells are reprogrammed to form embryonic stem like cells called induced pluripotent stem cells (IPSCs). Oral fibroblasts are able to form IPSCs in lab applicable for future use. However, the downside is that some of the transcription factors used is well known oncogenes. Viruses used in the technique have intrinsic risk in regard to cell transformation (14).

7-Oral epithelial stem cells are derived from basal layer of the epithelial oral mucosa. They are unipotent stem cells and possess clonogenicity. They can form highly stratified and well organized graft (9). Salivary gland stem cells derived from the stromal tissue of salivary glands. They are useful for regeneration of salivary gland damaged from irradiation and can be guided to osteogenic, chondrogenic and adipogenic differentiation (15). 


\section{Different Types of Tooth Derived Stem Cells}

1. Adipocytes: They have successfully been utilized to repair injure to the heart muscle caused by severe heart attack. There is initial data to point out that they can be used to treat cardiovascular diseases, orthopedic problems and spine, Crohn's disease, congestive heart failure and also used in plastic surgery.

2. Chondrocytes and osteoblasts: This type of stem cells has effectively been used to grow cartilages and bones suitable for transplant. They have also been utilized to develop intact teeth in animals.

3. Mesenchymal cells: These stem cells have successfully been used to restore spinal cord damage and to recall feelings and progress of movement in paralyzed patients. MSCs have the possibility to treat neuronal degenerative disorders such as Parkinson's diseases and Alzheimer's, cerebral palsy (16).

Collection, Isolation and Preservation of Dental Stem Cells Collection

The main source of dental stem cells is extracted teeth (Figure 1). A freshly extracted tooth is transferred into a carefully sealed vial containing transport solution and generally hypotonic phosphate buffered saline (PBS) solution. The vial placed into a thermette and carried into an insulated metal transport vessel. This maintain the sample in a hypothermic state during transportation and described as sustentation. The time from harvesting to arrival at the processing storage should not exceed 40 hours. Dental pulp stem cells samples are obtained from dental pulp tissue from extracted third molars, exfoliating/extracted deciduous teeth and teeth extracted for orthodontic treatment, trauma or periodontal disease. PDLSCs are obtained via scraping the mid third of the root in extracted teeth. SCAPs are isolated from the apical papilla tissue and can easily be removed from an extracted tooth with developing roots (17).

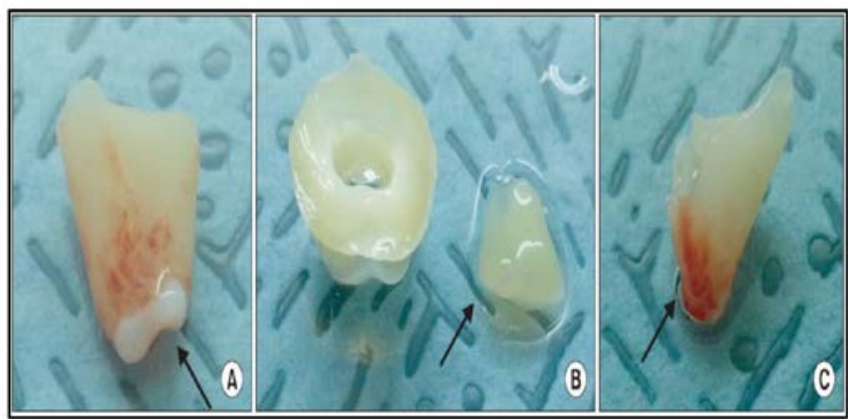

Figure (1):Human 3rd molars provide 3 different cell sources. A. Apical papilla from the developing root tip was gently separated from the surface of the root. B. Dental pulp was isolated from the cracked crown. C. The periodontal ligament was removed from the middle root surface.

\section{Isolation}

Almost every author uses a method of his own, which makes a detailed analysis of all experimental works impossible. Consequently, several studies need to be done in order to systematize laboratorial procedures. The methods most frequently used for DPSCs isolation are enzyme-digestion and explant

Alexandria Dental Journal. Volume 46 Issue 1 Section A outgrowth. Other methods like magnetic activated cell sorting and fluorescence activated cell sorting are available. Regardless of the chosen isolation method, common procedure dictates that the tooth surface is cleaned by washing it 3 times with PBS without $\mathrm{Ca} 2+$ and $\mathrm{Mg} 2+$. The next step is disinfection followed by another washing with PBS. Pulp tissue is isolated from the pulp chamber and placed into a sterile petri dish (18).

Enzyme-digestion method: Small pieces of pulp tissues are transferred into an enzyme solution, often collagenase for 30$60 \mathrm{~min}$ at $37^{\circ} \mathrm{C}$. Afterwards, large cell aggregates are removed and single-cell suspensions are obtained by passing cells through a cell strainer. Single cell suspensions are centrifuged for $5 \mathrm{~min}$ at room temperature. Suspensions are then seeded in culture dishes containing proliferation medium and incubated at $37^{\circ} \mathrm{C}$ in $5 \% \mathrm{CO} 2$. Culture medium can be substituted every 3 days until cell confluence is achieved. In explant outgrowth method; pulp tissue is placed in culture flask with proliferation medium and then incubated at $37^{\circ} \mathrm{C}$ in $5 \% \mathrm{CO} 2$. Medium must be changed after outgrowth is observed.

Additional method has been proposed. The main difference relies on the digestion of pulp tissues and fixing them under a cover slip in the medium. This method provided a greater in vitro expansion. Moreover, the cells were less damaged and were healthy enough to propagate longer in vitro than with other methods (19).

\section{Preservation}

Once the tissue samples are obtained, they are transferred under proper conditions to a tooth bank where they are stored. The approaches used for stem cell storage are cryopreservation and magnetic freezing. Cryopreservation is the process of preserving cells or whole tissues by cooling them at subzero temperatures. Liquid nitrogen vapor is used to preserve the cells at a temperature below $-150^{\circ} \mathrm{C}$. Cryopreservation of stem cells maintains the viability of these cells indefinitely. Magnetic freezing technology is referred to as cells ative system (CAS), which works on the principle of applying a weak magnetic field to water or cell tissue, which will lower the freezing point of that body by up to $6-7^{\circ} \mathrm{C}$. Using CAS system is much less expensive and more reliable than cryogenics (20).

Dental Stem Cells in Development and Repair of Dental and Oromaxillofacial Structures

Craniofacial Defects: Stem cells can be useful in the regeneration of bone and to correct large craniofacial defects due to cyst enucleation, tumor resection and trauma. Adipose derived stem cells were used to treat the defect of severe head injury. Autologous adipose stem cells were extracted from gluteal region along with iliac crest bone graft. This successful technique has given new rays of hope that adipose derived stem cells can be used for difficult reconstructive procedures (21)

Soft Tissue Reconstruction: The oromaxillofacial region is of paramount importance when there is significant loss of soft tissues during surgery or trauma. Various methods including graft and flap transfer have been tried that produced donor site morbidity. Human MSCs can turn into adipose cells when adipose cells with appropriate shaped scaffold can be used for reconstruction of soft tissues. Stem cells isolated from SCEDs have significantly promoted wound healing, proving deciduous teeth can be utilized for the treatment of chronic wounds. This application can be extended into the oromaxillofacial region to enhance wound healing (17). 
Periodontium: Regenerating the periodontium has always been a high priority in craniofacial regenerative biology. Due to the complex structure of the periodontium (consisting of hard and soft tissues: cementum, bone, periodontal ligament and gingiva), its complete regeneration would require a multipotent cell population. A study in periodontal defects in dogs demonstrated that transplantations of ex vivo expanded autologous mesenchymal stem cell can regenerate new cementum, alveolar bone, and periodontal ligament (22).

Dentin Regeneration: Migration of stem/progenitor cells to the site of injury for differentiation into a new generation of odontoblasts like cells. Reparative dentinogenesis and dentine bridge formation occur during pulp capping procedures. Exciting possibilities exist to exploit this aspect of regeneration, both in respect of maximizing recruitment of progenitor cells and also, perhaps through influencing the nature of the cell populations recruited (23).

Apexogenesis or Apexification: Regeneration of tissue into the apex of an immature permanent tooth may come from stem cells already residing in vital pulp tissue, the apical papilla, periodontal ligament or alveolar bone (10). Irreversible pulpal injury results in pulp necrosis and is commonly due to endodontic infection. In younger patients, where the possibility exists to retain some vital pulp tissue and allows continued root development, a conservative approach is desirable. When infection extends throughout the root canal system, endodontic treatment involyes the removal of remaining pulp tissue to the level of the developing root apex leading to loose physical connection with the apical papilla. Clinicians are guided by radiographs, apex locators, tactile sensation and reproducible drying points, but it is impossible to know where the pulp tissue terminates and if all pulpal cells are removed. Immature permanent teeth have a rich cellular and vascular supply and so DPSC and SCAP may survive disinfection and undergoing apexogenesis (24).

Tooth Regeneration: Different studies reported significant progress toward the creation of tissue-engineered embryonic tooth primordia (tooth buds) using cultured cells. Human tooth germ results from interactions between epithelial stem cell mass and neural crest derived mesenchymal stem cell mass during early embryonic development. Once embryonic oral epithelium and mesenchyme start interaction, the epithelial stem cells differentiate into ameloblasts; the MSCs differentiate into odontoblasts, fibroblasts and other cells; finally forming functional teeth (Figure 2). In order to regenerate a human tooth, two types of human stem cells are required: epithelial and MSCs $(25,26)$.

Regeneration of Various Tissues or Organs from Tooth Derived Stem Cells (Figure 3)

Osseous Regeneration: The DPSCs contain bone specific markers and exhibit an osteogenic differentiation profile. Upon differentiating into pre-osteoblasts, DPSCs deposit an extracellular matrix that eventually forms mineralized woven bone (12). Graziano et al., showed that CD34+DPSCs transplanted in the subcutaneous tissue of rats form a substantial number of bone nodules. DPSCs are considered one of the best candidates for bone regeneration (28). Consistently, the reconstruction of large-scale cranial bone defect was reported in non-immunocompromised rats. In a clinical study, bio-complexes prepared from DPSCs and collagen sponges were used in human mandible repair and Alexandria Dental Journal. Volume 46 Issue 1 Section A exhibited impressive results (29). On the other hand, the role of DPSCs in bone regeneration around dental implants was investigated, and the periosteal cells showed that DPSCs exhibit the highest osteogenic potential as a source for tissueengineered bone around titanium implants (30). Osseous regeneration using PDLSCs has been investigated. They form mineralized tissues with odontoblastic differentiation only under special environments, such as in the existence of intact dentin (27).

Neural Regeneration: Despite the limited amount of adult neural stem cell damage caused by harvesting cells, neural regeneration is considered imperative, since many nerve degenerations related diseases have no effective remedies and the sequelae are usually catastrophic. Thus, there have been tremendous attempts to identify appropriate stem cells with neural potentials. DPSCs exhibit markers of multi-potency, several of which are associated with spontaneous neural differentiation. Considering the origin of dental pulp tissue, it may not be surprising that DPSCs exhibit intrinsic neuro-glial characteristics and are capable of differentiating into both neural and vascular endothelial cells $(4,6)$. Regulation of the differentiation of DPSCs into different neuronal phenotypes was investigated at the molecular level. In addition, differentiated murine DPSCs were shown to form immature neuronal like networks (31).

Treatment of Myocardial Infarction: Despite recent advances in prevention and treatment, myocardial infarction remains one of the cardinal causes of mortality worldwide. The possibility of using DPSCs for the treatment of myocardial infarction was noted. For example, Gandia et al., (32) used human dental pulp stem cells in a rat myocardial infarction model, reporting an increase in the number of vessels and decrease in the size of infarction. A highly vasculogenic sub-fraction of DPSCs can be isolated from porcine dental pulp, which is similar to endothelial progenitor cells of dental pulp. Importantly, successful engraftment of these cells was observed upon transplanting this isolated cell population into the ischemic hind limb of mice (33). Therefore, DPSCs may represent an attractive stem cell source for tissue engineering and could be a treatment for inadequate angiogenesis such as chronic wounds, stroke, and myocardial infarction (27).

Hepatocyte Differentiation: Hepatocytes are the main cellular component of the liver, comprising $70-80 \%$ of the total liver mass. Ishkitiev et al., (34) were the first researchers who showed the differentiation potential of DPSCs into hepatocyte-like cells. This provided strong evidence to support a role for DPSCs in the treatment of irreversible liver disease, providing hope for a future cure.

Corneal Reproduction: Reconstruction of the cornea using DPSCs has been explored. A tissue-engineered sheet of DPSC was transplanted on the corneal bed and de-epithelialized human amniotic membrane covering was done. Healthy uniform corneal epithelium was formed after three months of healing. DPSCs isolated from third molars were also reported to be capable of differentiating into keratinocytes, which are cornea stroma cells. In addition to cornea reconstruction, human PDLSCs may also be directed towards retinal progenitors having competence for photoreceptor differentiation (35). 
Treatment of Diabetes: Diabetes is more and more increasing these days and becomes one of the most common chronic endocrinal diseases associated with pancreatic islet cell dysfunction. Not only type 1 but also type 2 diabetes may be successfully managed by transplantation of pancreatic islet cells. The differentiation potential of DPSCs into islet-like cell aggregates (ICAs) has been explored, with the results suggesting that in vitro cultured ICAs can release insulin and C-peptide in a glucose dependent manner. The transdifferentiation potential of human PDLSCs cultured into pancreatic islet cells has been demonstrated (36).

Miscellaneous: Differentiation of DPSCs into skeletal muscle has been reported for the treatment of muscular dystrophy (37). In addition, several studies have attempted to differentiate DPSCs into salivary gland cells (38).

\section{Future Perspectives}

Despite the changing trends along the available technological advances, regenerative medicine can be defined as it replaces or regenerates human cells, tissue, or organs, to restore or establish normal functions. Since there are a number of irreversible medical conditions that the current approaches cannot reverse, this field is more and more expanding with increasing interest.

Cancer Stem Cells (CSC): They are the new and potentially important targets for the therapy of oral squamous cell carcinoma. There is increasing evidence that the growth and spread of cancers is driven by a small subpopulation of CSCs. These are only cells that are capable of long term self-renewal and generation of the phenotypically diverse tumor cell population. Current failure of cancer therapies may be due to their lesser effect on potentially quiescent CSCs which remain vital and retain their full capacity to repopulate the tumor. Treatment strategies for the elimination of cancer therefore need to consider the consequences of the presence of CSCs. However, the development of new CSC targeted strategies is currently hindered by the lack of reliable markers for the identification of CSCs and the poor understanding of their behavior and fate determinants (39).

Cancer Treatment by Stem Cell Transplantation: The main purpose of stem cell transplantation in cancer treatment is to make it possible for patients to receive yery high doses of chemotherapy and/or radiation. High dose chemotherapy and radiation can severely damage or destroy bone marrow while killing cancer cells. Without healthy bone marrow, the body is no longer able to make the blood cells needed to prevent infection, bleeding, and carry oxygen. Stem cell transplants replace the stem cells destroyed by high dose cancer treatment allowing bone marrow to produce healthy cells (39).

\section{Stem Cell Banks}

Dental stem cells are a valuable source of stem cells and are found in teeth with healthy pulp. These teeth could be deciduous teeth, wisdom teeth and other permanent teeth. Presently, these teeth are being discarded as medical waste. A stem cell bank allows an individual the opportunity to preserve their biomaterial for future regenerative therapies (20). The banking of umbilical cord blood as a source of stem cells, which has been advocated for the past 15 years to parents of newborns. To date, there have been many lives saved through the transplantation of these stem cells. It was said. "Cryopreservation or banking of stem cells maintains the viability of these cells indefinitely". During cryopreservation, Alexandria Dental Journal. Volume 46 Issue 1 Section A the cells are put to sleep through a process called nitrification, in which the tissue is placed in liquid nitrogen at a temperature of -196 degrees Celsius. The cryopreservation process stops all cellular metabolism involving both cell growth and cell death. The cells preserved today can be applied to future regenerative therapies. There are many parents who did not have the opportunity to bank their children's umbilical cord blood. Stem cell banking allows these parents to save their children's accessible and valuable stem cells when they are undergoing a routine dental procedure (40).

\section{CONCLUSION}

Tooth derived stem cells represent a viable source of adult stem cells for regenerative medicine. The unique origin of these cells can contribute to the regeneration of various tissue types, although their characteristics are slightly different according to the isolation location. DPSCs are the most studied cell type among them and regeneration of tooth structures is the main topic. However, the tooth derived stem cells have shown to be a possible cell source for regeneration of non-tooth structures, such as bone, nerve, muscle, liver, and pancreas. Their application will expand along the technological advances although our current information and experience are not enough. Subsequent researches are expected to be done with their fascinating benefit as a readily available cell source in the future.

\section{REFERENCE}

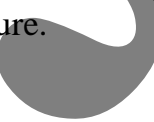

1. Egusa H, Sonoyama W, Nishimura M, Atsuta, Akiyama K. Stem cells in dentistry-part I: stem cell sources. J Prosthodont Res. 2012; 56:151-65.

2. Gardner RL. Stem cells: potency, plasticity and public perception. J/Anat. 2002; 200:277-82.

3. Ferreira LM, Mostajo-Radji MA. How induced pluripotent stem cells are redefining personalized medicine. Gene. 2013; 520:1-6.

4. Estrela C, de Alencar AH, Kitten GH, Vencio EF, Gava E. Mesenchymal stem cells in the dental tissues: perspectives for tissue regeneration. Braz Dent J. 2011; 22:91-8.

Xiao L, Nasu M. From regenerative dentistry to regenerative medicine: progress, challenges, and potential applications of oral stem cells. Stem Cells Cloning. 2014; 7:89-99.

6. Li D, Zou XY, El-Ayachi I, Romero LO, Yu Z, IglesiasLinares A, et al. Human dental pulp stem cells and gingival mesenchymal stem cells display action potential capacity in vitro after neurogenic differentiation. Stem Cell Rev Rep. 2019; 15:67-81.

7. Gronthos S, Mankani M, Brahim J, Robey PG, Shi S. Postnatal human dental pulp stem cells (DPSCs) in vitro and in vivo. Proc Natl Acad Sci U S A. 2000; 97:13625-30.

8. Kerkis I, Caplan AI: Stem cells in dental pulp of deciduous teeth. Tissue Eng Part B Rev. 2012; 18:129-38.

9. Hassan NT, AbdelAziz NA. Oral mucosal stem cells, human immature dental pulp stem cells and hair follicle bulge stem cells as adult stem cells able to correct limbal stem cell deficiency. Curr Stem Cell Res Ther. 2018; 13:356-61.

10. Shilpa PS, Kaul R, Sultana N, Bhat S. Stem cells: boon to dentistry and medicine. Dent Res J (Isfahan). 2013; 10:149-54. 
11. Morsczeck C. Molecular mechanisms in dental follicle precursor cells during the osteogenic differentiation. Histol Histopathol. 2015; 30:1161-9.

12. Colangeli W, Riccelli U, Giudice A, Barca I, Caruso D, Novembre $\mathrm{D}$, et al. Jaw bones regeneration using mesenchymal stem cells. A single-center experience. Ann Ital Chir. 2018; 89:20-3.

13. Tata PR, Mou H, Pardo-Saganta A, Zhao R, Prabhu M, Law BM, et al. Dedifferentiation of committed epithelial cells into stem cells in vivo. Nature. 2013; 503:218-23.

14. Mitsuzawa S, Ikeguchi R, Aoyama T, Ando M, Takeuchi $\mathrm{H}$, Yurie $\mathrm{H}$, et al. Induced pluripotent stem cell-derived mesenchymal stem cells prolong hind limb survival in a rat vascularized composite allotransplantation model. Microsurgery. 2019; 10:30507.

15. Pringle S, Maimets M, van der Zwaag M, Stokman MA, van Gosliga D, Zwart E, et al. Human salivary gland stem cells functionally restore radiation damaged salivary glands. Stem Cells. 2016; 34:640-52.

16. Petrovic V, Stefanovic V. Dental tissue--new source for stem cells. Scientific World Journal. 2009; 14:1167-77.

17. Bansal R, Jain A. Current overview on dental stem cells applications in regenerative dentistry. J Nat Sci Biol Med. 2015; 6:29-34.

18. Derakhshani A, Raoof M, Dabiri S, Farsinejad AR, Gorjestani H, Yaghoobi MM, et al. Isolation and evaluation of dental pulp stem cells from teeth with advanced periodontal disease. Arch Iran Med. 2015; 18:211-7.

19. Raoof M, Yaghoobi MM, Derakhshani A, Kamal-Abadi AM, Ebrahimi B, Abbasnejad $\mathrm{M}$, et al. A modified efficient method for dental pulp stem cell isolation. Dent Res J (Isfahan). 2014; 11:244-50.

20. Arora V, Arora P, Munshi AK. Banking stem cells from human exfoliated deciduous teeth (SHED): saving for the future. J Clin Pediatr Dent. 2009; 33:289-94.

21. San-Marina S, Sharma A, Voss SG, Janus JR, Hamilton GS 3rd. Assessment of scaffolding properties for chondrogenic differentiation of adipose-derived mesenchymal stem cells in nasal reconstruction. JAMA Facial Plast Surg. 2017; 19:108-14.

22. Yu X, Ge S, Chen S, Xu Q, Zhang J, Guo H, et al. Human gingiva-derived mesenchymal stromal cells contribute to periodontal regeneration in beagle dogs. Cells Tissues Organs. 2013; 198:428-37.

23. Kim SG. Biological molecules for the regeneration of the pulp-dentin complex. Dent Clin North Am. 2017; 61:127-41.

24. Chueh LH, Huang GT. Immature teeth with periradicular periodontitis or abscess undergoing apexogenesis: a paradigm shift. J Endod. 2006; 32:1205-13.

25. Bluteau G, Luder HU, De Bari C, Mitsiadis TA. Stem cells for tooth engineering. Eur Cell Mater. 2008; 16:1-9.

26. Chen Y, Yu Y, Chen L, Ye L, Cui J, Sun Q, et al. Human umbilical cord mesenchymal stem cells: a new therapeutic option for tooth regeneration. Stem Cells Int. 2015; 2015:549432.

27. Rajendran R, Gopal S, Masood H, Vivek P, Deb K.
Regenerative potential of dental pulp mesenchymal stem cells harvested from high caries patient's teeth. J Stem Cells. 2013; 8:25-41.
28. Graziano A, d'Aquino R, Laino G, Proto A, Giuliano MT, Pirozzi G, et al. Human CD34+ stem cells produce bone nodules in vivo. Cell Prolif. 2008; 41:1-11.

29. d'Aquino R, De Rosa A, Lanza V, Tirino V, Laino L, Graziano A, et al. Human mandible bone defect repair by the grafting of dental pulp stem/progenitor cells and collagen sponge biocomplexes. Eur Cell Mater. 2009; 18:75-83.

30. Ito K, Yamada Y, Nakamura S, Ueda M. Osteogenic potential of effective bone engineering using dental pulp stem cells, bone marrow stem cells, and periosteal cells for osseointegration of dental implants. Int J Oral Maxillofac Implants. 2011; 26:947-54.

31. Ellis KM, O'Carroll DC, Lewis MD, Rychkov GY, Koblar SA. Neurogenic potential of dental pulp stem cells isolated from murine incisors. Stem Cell Res Ther. 2014; 5:30.

32. Gandia C, Arminan A, Garcia-Verdugo JM, Lledo E, Ruiz A, Minana MD, et al. Human dental pulp stem cells improve left ventricular function, induce angiogenesis, and reduce infarct size in rats with acute myocardial infarction. Stem Cells. 2008; 26:638- 45.

33. Iohara K, Zheng L, Wake H, Ito M, Nabekura J, Wakita H, et al. A novel stem cell source for vasculogenesis in ischemia: subfraction of side population cells from dental pulp. Stem Cells. 2008; 26:2408-18.

34. Ishkitiev N, Yaegaki K, Imai T, Tanaka T, Fushimi N, Mitev $\mathrm{V}$, et al. Novel management of acute or secondary biliary liver conditions using hepatically differentiated human dental pulp cells. Tissue Eng Part A. 2015; 21:586-93.

35. Syed-Picard FN, Du Y, Lathrop KL, Mann MM, Funderburgh ML, Funderburgh JL. Dental pulp stem cells: a new cellular resource for corneal stromal regeneration. Stem Cells Transl Med. 2015; 4:276-85.

36. Lee JS, An SY, Kwon IK, Heo JS. Transdifferentiation of human periodontal ligament stem cells into pancreatic cell lineage. Cell Biochem Funct. 2014; 32:605-11.

37. Yang R, Chen M, Lee CH, Yoon R, Lal S, Mao JJ. Clones of ectopic stem cells in the regeneration of muscle defects in vivo. PLoS One. 2010; 5:e13547.

38. Yamamura Y, Yamada $H$, Sakurai T, Ide F, Inoue $H$, Muramatsu $\mathrm{T}$, et al. Treatment of salivary gland hypofunction by transplantation with dental pulp cells. Arch Oral Biol. 2013; 58:935-42.

39. Vlashi E, Pajonk F. Cancer stem cells, cancer cell plasticity and radiation therapy. Semin Cancer Biol. 2015; 31:28-35.

40. Aly LA. Stem cells: Sources, and regenerative therapies in dental research and practice. World J Stem Cells. 2015; 7:1047-53. 